\title{
Low-mass lepton pair production at large transverse momentum
}

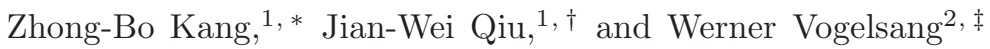 \\ ${ }^{1}$ Department of Physics and Astronomy, Iowa State University, Ames, IA 50011, USA \\ ${ }^{2}$ Physics Department, Brookhaven National Laboratory, Upton, NY 11973, USA
}

(Dated: November 8, 2018)

\begin{abstract}
We study the transverse momentum distribution of low-mass lepton pairs produced in hadronic scattering, using the perturbative QCD factorization approach. We argue that the distribution at large transverse momentum, $Q_{T} \gg Q$, with the pair's invariant mass $Q$ as low as $Q \sim \Lambda_{\mathrm{QCD}}$, can be systematically factorized into universal parton-to-lepton pair fragmentation functions, parton distributions, and perturbatively calculable partonic hard parts evaluated at a short distance scale $\sim \mathcal{O}\left(1 / Q_{T}\right)$. We introduce a model for the input lepton pair fragmentation functions at a scale $\mu_{0} \sim 1 \mathrm{GeV}$, which are then evolved perturbatively to scales relevant at RHIC. Using the evolved fragmentation functions, we calculate the transverse momentum distributions in hadronhadron, hadron-nucleus, and nucleus-nucleus collisions at RHIC. We also discuss the sensitivity of the transverse momentum distribution of low-mass lepton pairs to the gluon distribution.
\end{abstract}

PACS numbers: 12.38.Bx,12.39.St,13.85.Qk,14.70.Dj

\section{INTRODUCTION}

Hadronic processes producing a high-transverse-momentum $\left(Q_{T}\right)$ photon play a fundamental role in High-Energy Physics. They offer possibilities to explore QCD, probing for example the structure of the interacting hadrons or testing the predictive power of QCD calculations. They are intimately involved in many signals (and their backgrounds) for New Physics, and may also serve as important probes of the properties of the strongly-interacting matter produced in heavy-ion collisions. Their "point-like" QED coupling to quarks makes photons particularly suited for these applications.

One can distinguish two types of high- $Q_{T}$ photon signals, depending on whether the produced photons are real $\left(Q^{2}=0\right)$, or virtual $\left(Q^{2}>0\right)$. The first case corresponds to the "classic" prompt-photon situation [1]. In the second case, the photon could subsequently decay into a lepton pair $\ell^{+} \ell^{-}$, which can be detected. This case thus corresponds to a "Drell-Yan" type situation, with a lepton pair at high $Q_{T}[2,[3,4,4]$.

Both types have their virtues and disadvantages. Electromagnetic probes of QCD hard scattering are generally relatively rare compared to hadronic ones such as jets. At a given $Q_{T}$, real photons are much more copious than lepton pairs from virtual photon decay, as the latter suffer from much lower rates, both due to an additional power of the electromagnetic coupling $\alpha_{\mathrm{em}}$ and to a phase space suppression. On the other hand, one would intuitively expect that virtual photons could both give experimentally cleaner signals and be theoretically more tractable. Real photons may have large backgrounds from $\pi^{0}$ decay or may be faked in the detector by neutral hadrons, in particular toward lower $Q_{T}$. While these problems can be addressed in part by applying an isolation cut to the photon signal, for lepton pairs they will not be there in the first place. On the theoretical side, complications in the calculation of the cross section that are present at $Q^{2} \approx 0$ may be alleviated if $Q^{2} \gg \Lambda_{\mathrm{QCD}}^{2}$. These complications are related to the presence of a "fragmentation" (or, "bremsstrahlung") contribution to the real-photon cross section, which arises from the observed photon being produced in jet fragmentation [1]. Since this contribution involves non-perturbative parton-to-photon fragmentation functions [6, 7, 8], about which there is only limited knowledge, it leads to an uncertainty in the theoretical predictions. When, however, $Q$ is much larger than typical non-perturbative scales in $\mathrm{QCD}, Q_{T} \gg Q \gg \Lambda_{\mathrm{QCD}}$, the fragmentation part can be calculated in factorized perturbation theory, as was shown in [4, 5], which allows rather precise theoretical estimates of the cross section.

Clearly, the situation $Q_{T} \gg Q \gg \Lambda_{\mathrm{QCD}}$ is ideal from a theoretical point of view but tough to realize in experiment, due to the low rates of lepton pairs with these kinematics. In the present paper, we investigate the photon cross section in the regime $Q_{T} \gg Q \sim \Lambda_{\mathrm{QCD}}$. Our study is very much motivated by measurements performed for these kinematics [9] by the PHENIX collaboration at the Relativistic Heavy Ion Collider (RHIC) at Brookhaven National Laboratory

\footnotetext{
*Electronic address: kangzb@iastate.edu

${ }^{\dagger}$ Electronic address: jwq@iastate.edu

‡Electronic address: vogelsan@quark.phy.bnl.gov
} 
(BNL). Choosing lepton pairs with $100 \leq Q \leq 300 \mathrm{MeV}$ allows PHENIX to extend their photon measurements to lower transverse momenta, $Q_{T} \leq 5 \mathrm{GeV}$, than possible for real prompt photons for which the background from neutral hadrons becomes overwhelming. Thus the improvement in systematic uncertainties offered by the low-mass lepton pairs potentially outweighs the loss in statistics. At higher $Q_{T} \gtrsim 5 \mathrm{GeV}$, it becomes eventually more beneficial to consider real photons. However, much of the interesting physics in heavy-ion collisions or (polarized) $p p$ scattering takes place in the region $Q_{T} \leq 5 \mathrm{GeV}$.

When $Q \sim \Lambda_{\mathrm{QCD}}$, the fragmentation contribution to the cross section will no longer be completely perturbative as it was for $Q \gg \Lambda_{\mathrm{QCD}}$. However, we will argue that, like for real photons, the hadronic cross section for producing high transverse momentum lepton pairs with low invariant mass can be systematically factorized into universal fragmentation functions, parton distributions, and perturbatively calculable partonic hard parts evaluated at a distance scale $\sim \mathcal{O}\left(1 / Q_{T}\right)$. We note that this is similar in spirit to what is typically done in the corresponding "space-like" case of the structure functions of virtual photons [10], which received a lot of attention at HERA [11]. Thus, our main conclusion will be that the lepton-pair cross section in this kinematic regime can be treated in a similar fashion, and with similar rigor, as the real-photon one, which makes the efforts to use it experimentally as a means to access lower $Q_{T}$ very worthwhile. We will also discuss the connection between the very low mass dilepton pair and direct real-photon production. We note that there has been an earlier theoretical study of the dilepton cross section in this kinematic regime [2]. Our study goes beyond that work, particularly by providing a more complete treatment of the fragmentation contribution and more extensive phenomenological studies.

We will introduce a model for the input lepton pair fragmentation functions at a scale $\mu_{0} \sim 1 \mathrm{GeV}$, which are then evolved perturbatively to scales relevant at RHIC. Using the evolved fragmentation functions, we calculate the transverse momentum distributions of lepton pairs in proton-proton collisions at $\sqrt{s}=200 \mathrm{GeV}$. We also discuss the case of nuclear collisions at RHIC, and investigate the sensitivity of high- $Q_{T}$ low mass dileptons to the nuclear gluon distribution.

The rest of our paper is organized as follows. In Sec. II we review the perturbative QCD factorization for the Drell-Yan cross section when $Q_{T} \gg Q \gg \Lambda_{\mathrm{QCD}}$, to set up the notation and terminology. In Sec. [II] we study the asymptotic limit of the factorized cross section as $Q \rightarrow 0$. We demonstrate that all perturbative short-distance parts of the factorized cross section are finite and can be expanded as power series in $Q^{2} / Q_{T}^{2}$. All non-perturbative physics present when $Q \sim \Lambda_{\mathrm{QCD}}$ is systematically included in new non-perturbative "parton-to-low-mass-dilepton" fragmentation functions. We introduce our model for these fragmentation functions at a scale $\mu_{0}=1 \mathrm{GeV}$. In Sec. IV] we use the perturbatively evolved fragmentation functions to obtain predictions for $p p$ scattering at RHIC. We also study the nuclear dependence of low-mass dilepton transverse momentum distributions, and their role for extracting nuclear gluon distributions. Finally, Section $\nabla$ presents our summary and conclusions.

\section{LEPTON PAIR PRODUCTION WITH $Q_{T} \gg Q \gg \Lambda_{\mathrm{QCD}}$}

The cross section for Drell-Yan type inclusive lepton pair production in hadronic collisions, $A\left(P_{A}\right)+B\left(P_{B}\right) \rightarrow$ $\gamma^{*}\left(\rightarrow \ell^{+} \ell^{-}(Q)\right)+X$, as sketched in Fig. 1 can be expressed in terms of the cross section for producing a virtual photon that decays into the observed lepton pair:

$$
\frac{d \sigma_{A B \rightarrow \ell^{+} \ell^{-}(Q) X}}{d Q^{2} d Q_{T}^{2} d y}=\left(\frac{\alpha_{\mathrm{em}}}{3 \pi Q^{2}}\right) \sqrt{1-\frac{4 m_{\ell}^{2}}{Q^{2}}}\left(1+\frac{2 m_{\ell}^{2}}{Q^{2}}\right) \frac{d \sigma_{A B \rightarrow \gamma^{*}(Q) X}}{d Q_{T}^{2} d y},
$$

where the variables $Q, Q_{T}$, and $y$ are the invariant mass, transverse momentum, and rapidity of the virtual photon, respectively. $m_{\ell}$ is the lepton mass, and the symbol $X$ stands for an inclusive sum over final states that recoil against the virtual photon. The overall factor on the right-hand-side of Eq. (11) arises from the integration over the pair's angular distribution, $\sqrt{1-4 m_{\ell}^{2} / Q^{2}}$ representing the mass threshold for producing the pair.

When both physically measured momentum scales $Q$ and $Q_{T}$ are much larger than $\Lambda_{\mathrm{QCD}}$, the cross section for producing the virtual photon can be factored systematically in QCD perturbation theory as [12]

$$
\frac{d \sigma_{A B \rightarrow \gamma^{*}(Q) X}}{d Q_{T}^{2} d y}=\sum_{a, b} \int d x_{1} f_{a}^{A}\left(x_{1}, \mu\right) \int d x_{2} f_{b}^{B}\left(x_{2}, \mu\right) \frac{d \hat{\sigma}_{a b \rightarrow \gamma^{*}(Q) X}^{\mathrm{Pert}}}{d Q_{T}^{2} d y}\left(x_{1}, x_{2}, Q, Q_{T}, y ; \mu\right),
$$

where the sum $\sum_{a, b}$ runs over all parton flavors, $x_{1}$ and $x_{2}$ are the partons' momentum fractions, and $f_{a}^{A}$ and $f_{b}^{B}$ are the corresponding parton distributions. $\mu$ represents the renormalization and factorization scale; it is of the order of the energy exchange in the reaction: $\mu \sim \sqrt{Q^{2}+Q_{T}^{2}}$. The function $d \hat{\sigma}_{a b \rightarrow \gamma^{*}(Q) X}^{\text {Pert }} / d Q_{T}^{2} d y$ in Eq. (2) represents the short-distance physics of the collision and is calculable perturbatively in terms of a power series in $\alpha_{s}(\mu)$. 


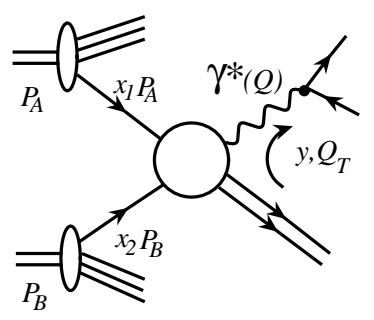

FIG. 1: Sketch for the "Drell-Yan type" hadronic production of a lepton pair via the decay of a virtual photon.

Beyond the leading order in $\alpha_{s}$, when $Q_{T} \gg Q$, the short-distance perturbative functions $d \hat{\sigma}_{a b \rightarrow \gamma^{*}(Q) X}^{\text {Pert }} / d Q_{T}^{2} d y$ in Eq. (2) can receive large high order corrections in powers of $\alpha_{s} \ln \left(Q_{T}^{2} / Q^{2}\right)$ which are caused by the radiation of partons along the direction of the low mass lepton pair. Such large logarithmic corrections can be systematically resummed into the parton-to-virtual photon fragmentation functions, $D_{f \rightarrow \gamma^{*}}$ [4]. The perturbative series for the $d \hat{\sigma}_{a b \rightarrow \gamma^{*}(Q) X}^{\text {Pert }} / d Q_{T}^{2} d y$ can therefore be re-organized into two terms as [5],

$$
\begin{aligned}
\frac{d \hat{\sigma}_{a b \rightarrow \gamma^{*}(Q) X}^{\mathrm{Pert}}}{d Q_{T}^{2} d y}\left(x_{1}, x_{2}, Q, Q_{T}, y ; \mu\right) & =\frac{d \hat{\sigma}_{a b \rightarrow \gamma^{*}}^{\mathrm{Dir}}(Q) X}{d Q_{T}^{2} d y}\left(x_{1}, x_{2}, Q, Q_{T}, y ; \mu, \mu_{F}\right) \\
& +\frac{d \hat{\sigma}_{a b \rightarrow \gamma^{*}(Q) X}^{\mathrm{Frag}}}{d Q_{T}^{2} d y}\left(x_{1}, x_{2}, Q, Q_{T}, y ; \mu, \mu_{F}\right),
\end{aligned}
$$

where the superscripts "Dir" and "Frag" represent the "direct" and the "fragmentation" contribution, respectively. The latter includes the perturbative fragmentation logarithms and can be further factorized as [4],

$$
\frac{d \hat{\sigma}_{a b \rightarrow \gamma^{*}(Q) X}^{\mathrm{Frag}}}{d Q_{T}^{2} d y}=\sum_{c} \int \frac{d z}{z^{2}}\left[\frac{d \hat{\sigma}_{a b \rightarrow c X}}{d p_{c_{T}}^{2} d y}\left(x_{1}, x_{2}, p_{c}=\hat{Q} / z ; \mu_{F}\right)\right] D_{c \rightarrow \gamma^{*}}\left(z, \mu_{F}^{2} ; Q^{2}\right),
$$

where the four-momentum $\hat{Q}^{\mu}$ is defined to be $Q^{\mu}$ at $Q^{2}=0$, corresponding to the approximation $Q^{2} \ll Q_{T}^{2}$ made for the fragmentation piece, and where $d \hat{\sigma}_{a b \rightarrow c X} / d p_{c_{T}}^{2} d y$ is a short-distance hard part for partons of flavors $a$ and $b$ to produce a parton of flavor $c$ and momentum $p_{c}=\hat{Q} / z$. The fragmentation contribution in Eq. (4) shares the typical two-stage generic pattern of the fragmentation production of a single particle at large transverse momentum $Q_{T}$ (e.g., a hadron of mass $M_{h} \ll Q_{T}$, or a real photon): the production of an on-shell parton of flavor $c$ at the distance scale $1 / Q_{T}$, convoluted with a fragmentation function that includes the leading logarithmic contributions from the "running" of the distance scale from $1 / \mu_{F} \sim 1 / Q_{T}$ to $1 / \mu_{F 0} \sim 1 / Q$. When $Q \gg \Lambda_{\mathrm{QCD}}$, the parton-to-virtual photon fragmentation function $D_{f \rightarrow \gamma^{*}}$ is perturbative, and so is the whole resummed fragmentation contribution, $\hat{\sigma}^{\text {Frag }}$ [4].

The direct contribution in Eq. (3) is perturbatively calculable in a power series of $\alpha_{s}$. The coefficient of the term at $n$-th power in $\alpha_{s}$ is given by [ [5] ,

$$
\begin{aligned}
& \frac{d \hat{\sigma}_{a b \rightarrow \gamma^{*}(Q) X}^{\operatorname{Dir}(n)}}{d Q_{T}^{2} d y}\left(x_{1}, x_{2}, Q, Q_{T}, y ; \mu, \mu_{F}\right) \\
& \quad \equiv \frac{d \hat{\sigma}_{a b \rightarrow \gamma^{*}(Q) X}^{\operatorname{Pert}(n)}}{d Q_{T}^{2} d y}\left(x_{1}, x_{2}, Q, Q_{T}, y ; \mu\right)-\frac{d \hat{\sigma}_{a b \rightarrow \gamma^{*}(Q) X}^{\operatorname{Asym}(n)}}{d Q_{T}^{2} d y}\left(x_{1}, x_{2}, Q, Q_{T}, y ; \mu, \mu_{F}\right),
\end{aligned}
$$

where $\hat{\sigma}^{\text {Pert(n) }}$ is the corresponding $n$-th order coefficient of $\hat{\sigma}^{\text {Pert }}$ in Eq. (2), calculated in conventional fixed-order QCD perturbation theory. The superscript "Asym" refers to an "asymptotic" contribution, which is simply the perturbative expansion of the fragmentation contribution, $\hat{\sigma}^{\text {Frag }}$ in Eq. (4) to the same $n$-th power in $\alpha_{s}$, including expansion of the resummed fragmentation function. The asymptotic contribution cancels the fragmentation logarithms in the perturbative contribution, $\hat{\sigma}^{\text {Pert }(n)}$, order-by-order in powers of $\alpha_{s}$. Therefore, the direct contribution is free of the large fragmentation logarithms, while it still keeps some information about the physics between the scales $Q_{T}$ and $Q$ from non-logarithmic terms, such as powers of $Q^{2} / Q_{T}^{2}$. These non-logarithmic terms in the direct contribution enable the new re-organized factorization formalism to cover a wide range of kinematics and to have a smooth transition from the low $Q_{T}$ to the high $Q_{T}$ region.

The separation between the direct and the fragmentation contribution in Eq. (3) depends on the definition of the parton-to-virtual photon fragmentation functions. A definition or a scheme choice for the fragmentation functions 
uniquely fixes the asymptotic contribution, which then fixes the direct contribution, in such a way as to render the sum $\hat{\sigma}^{\text {Pert }}$ independent of the adopted scheme.

The resummation of the fragmentation logarithms into the parton-to-virtual photon fragmentation functions is achieved by solving the inhomogeneous evolution equations [4],

$$
\begin{aligned}
\mu_{F}^{2} \frac{d}{d \mu_{F}^{2}} D_{c \rightarrow \gamma^{*}}\left(z, \mu_{F}^{2} ; Q^{2}\right) & =\left(\frac{\alpha_{\mathrm{em}}}{2 \pi}\right) \gamma_{c \rightarrow \gamma^{*}}\left(z, \mu_{F}^{2}, \alpha_{s} ; Q^{2}\right) \\
& +\left(\frac{\alpha_{s}}{2 \pi}\right) \sum_{d} \int_{z}^{1} \frac{d z^{\prime}}{z^{\prime}} P_{c \rightarrow d}\left(\frac{z}{z^{\prime}}, \alpha_{s}\right) D_{d \rightarrow \gamma^{*}}\left(z^{\prime}, \mu_{F}^{2} ; Q^{2}\right),
\end{aligned}
$$

where $c, d=q, \bar{q}, g$. The ambiguity in defining the fragmentation function is connected to the choice of the fragmentation scale, $\mu_{F}$, and the removal of the perturbative ultraviolet divergence in deriving the evolution kernels. In Eq. (6), the evolution kernels $P_{c \rightarrow d}$ are evaluated at a single hard scale, $\mu_{F}$, and can be calculated perturbatively as a power series in $\alpha_{s}$. The inhomogeneous term in the evolution equations can also be calculated perturbatively, and in general it has power correction terms of the form $Q^{2} / \mu_{F}^{2}$, due to the virtuality of the photon $Q^{2}$. In the invariant mass cut-off scheme [4], the lowest order quark-to-virtual photon evolution kernel is given by,

$$
\gamma_{q \rightarrow \gamma^{*}}^{(0)}\left(z, k^{2} ; Q^{2}\right)=e_{q}^{2}\left[\frac{1+(1-z)^{2}}{z}-z\left(\frac{Q^{2}}{z k^{2}}\right)\right] \theta\left(k^{2}-\frac{Q^{2}}{z}\right),
$$

where $k^{2}$ is the invariant mass of the parent quark and is identified as $\mu_{F}^{2}$, and where the $\theta$-function is a consequence of the mass threshold. The gluon-to-virtual-photon evolution kernel vanishes at the lowest order

$$
\gamma_{g \rightarrow \gamma^{*}}^{(0)}\left(z, k^{2} ; Q^{2}\right)=0
$$

because the gluon does not interact directly with the virtual photon. The choice of factorization scheme is not unique. But, some choices, such as the modified minimum subtraction $(\overline{\mathrm{MS}})$ scheme, may not respect the mass threshold when $Q^{2} \neq 0$ and lead to negative fragmentation functions [4, 13]. QCD corrections to the lowest order parton-to-virtual-photon splitting function $\gamma_{c \rightarrow \gamma^{*}}^{(0)}$ can be evaluated in principle order-by-order in $\alpha_{s}$.

If $Q \gg \Lambda_{\mathrm{QCD}}$, the parton-to-virtual photon fragmentation functions are completely perturbative. The input distributions for the evolution equation in Eq. (6) are given by

$$
D_{c \rightarrow \gamma^{*}}\left(z, \mu_{F}^{2} \leq Q^{2} / z ; Q^{2}\right)=0
$$

for any flavor $c$, if we choose the invariant mass cut-off scheme for the fragmentation functions [4, 5].

We conclude this section by summarizing the above equations and writing down a compact expression for the hadronic dilepton production cross section at high transverse momentum:

$$
\begin{aligned}
\frac{d \sigma_{A B \rightarrow \ell^{+} \ell^{-}(Q) X}}{d Q^{2} d Q_{T}^{2} d y} & =\left(\frac{\alpha_{\mathrm{em}}}{3 \pi Q^{2}}\right) \sqrt{1-\frac{4 m_{\ell}^{2}}{Q^{2}}}\left(1+\frac{2 m_{\ell}^{2}}{Q^{2}}\right) \\
& \times\left[\sum_{a, b} f_{a}^{A} \otimes f_{b}^{B} \otimes \frac{d \hat{\sigma}_{a b \rightarrow \gamma^{*}}^{\text {Dir }}(Q) X}{d Q_{T}^{2} d y}+\sum_{a, b, c} f_{a}^{A} \otimes f_{b}^{B} \otimes \frac{d \hat{\sigma}_{a b \rightarrow c X}}{d p_{c_{T}}^{2} d y} \otimes D_{c \rightarrow \gamma^{*}}\right]
\end{aligned}
$$

where $\otimes$ represents the convolution over parton momentum fractions. We stress that when both $Q_{T}$ and $Q$ are perturbative scales, the factorization into the direct and fragmentation contribution is merely a re-organization of $\hat{\sigma}^{\text {Pert }}$ with the fragmentation logarithms resummed to all orders. The re-organized perturbative expansion in Eq. (10) is perturbatively reliable for the full kinematic regime $Q_{T} \sim Q$ to $Q_{T} \gg Q$.

\section{LEPTON PAIR PRODUCTION AT $Q_{T} \gg Q \gtrsim \Lambda_{\mathrm{QCD}}$}

In this section, we examine the validity of the factorized formula in Eq. (10) when the invariant mass of the lepton pair decreases to $Q \gtrsim \Lambda_{\mathrm{QCD}}$.

The short-distance hard parts for the direct and the fragmentation contribution, $d \hat{\sigma}_{a b \rightarrow \gamma^{*}}^{\text {Dir }(Q) X} / d Q_{T}^{2} d y$ and $d \hat{\sigma}_{a b \rightarrow c X} / d p_{c_{T}}^{2} d y$ in Eq. (10), are evaluated at the distance scales $\sim \mathcal{O}\left(1 / Q_{T}\right)$ and $\sim \mathcal{O}\left(1 / p_{c_{T}}\right) \sim \mathcal{O}\left(1 / Q_{T}\right)$, respectively. Both hard parts are not sensitive to the dynamics at the scale $Q$ and are infrared safe perturbatively, even as $Q \rightarrow 0$. 


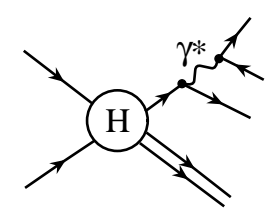

(a)

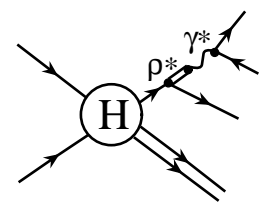

(b)

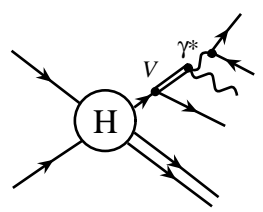

(c)

FIG. 2: Partonic scattering amplitudes for producing a large $Q_{T}$ lepton pair of very low invariant mass $Q$ by (a) a QED interaction via a virtual photon, (b) hadronic production of a virtual $\rho$ meson which then fluctuates into a virtual photon, or (c) a vector boson decay into a virtual photon plus unobserved final state.

However, when $Q$ decreases to $1 \mathrm{GeV}$ and below, there are significant non-perturbative sources to produce the low mass lepton pairs. Other than the Drell-Yan mechanism, or the QED production via a virtual photon shown in Fig. 2(a), lepton pairs can also be produced by the conversion of a virtual intermediate $\rho$ meson, as shown in Fig. 2(b), as well as in the decay of a light mass vector meson $V$, see Fig. 2(c). Since the vector meson is unlikely to be produced at the distance scale of $\mathcal{O}\left(1 / Q_{T}\right)$, these new channels of dilepton production are only relevant to the fragmentation contribution, and more precisely, to the fragmentation functions. That is, the fragmentation contribution to the production of very low-mass lepton pairs can still be factorized in the same way as that for producing a real photon or a single hadron at large transverse momentum, as indicated in Fig. 3. Therefore, we expect the QCD factorized formalism for low-mass dilepton production at high transverse momentum in Eq. (10) to be valid even when the invariant mass of the lepton pairs is as low as $100 \leq Q \leq 300 \mathrm{MeV}$, as considered at RHIC [9]. When the dilepton's invariant mass $Q$ is of the order of $\Lambda_{\mathrm{QCD}}$, the only change to Eq. (10) is that the parton-to-virtual photon fragmentation function, $D_{c \rightarrow \gamma^{*}}$, is no longer fully perturbative. The predictive power of the factorized formalism in Eq. (10) will rely on the universality of the parton-to-virtual photon fragmentation functions.
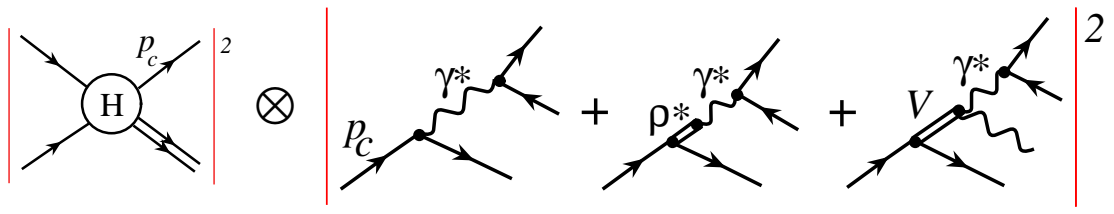

FIG. 3: Sketch for the leading-power factorized fragmentation contribution to the production of low-mass lepton pairs at large transverse momentum.

The parton-to-virtual photon (or lepton pair) fragmentation functions are defined in terms of matrix elements of gauge invariant non-local operators [4]. The resummation of the perturbative fragmentation logarithms into the fragmentation functions is still achieved by solving the same inhomogeneous evolution equations as in Eq. (6), except that the "natural" boundary condition in Eq. (9) needs to be replaced by non-perturbative input fragmentation functions at an initial scale, say, $\mu_{0} \sim 1 \mathrm{GeV}$. The inhomogeneous term in the evolution equations is still needed because of the perturbative QED production of the lepton pair via a virtual photon, as shown in Fig. 2(a).

In principle, the input fragmentation functions should be universal and determined by experimental data, like the fragmentation functions for producing a real photon or a single hadron. In order to estimate the size of the production rate of low mass lepton pairs at high transverse momentum, we propose a model for the input fragmentation functions with a minimal number of parameters, which we hope will serve as an initial step in determining the non-perturbative fragmentation functions.

The "natural" boundary condition in Eq. (9) is a consequence of the pure Drell-Yan production of lepton pairs via a single intermediate virtual photon, which does not involve any intermediate hadronic meson states, as shown in Fig. 2(a). With the additional non-perturbative sources of lepton pair production, like those shown in Fig. 2(b) and (c), we assume the following form of the non-perturbative input fragmentation functions at the scale $\mu_{0}=1 \mathrm{GeV}$ :

$$
D_{f \rightarrow \ell^{+} \ell^{-}(Q)}\left(z, \mu_{0}^{2} ; Q^{2}\right)=\left(\frac{\alpha_{\mathrm{em}}}{3 \pi Q^{2}}\right) \sqrt{1-\frac{4 m_{\ell}^{2}}{Q^{2}}}\left(1+\frac{2 m_{\ell}^{2}}{Q^{2}}\right) D_{f \rightarrow \gamma^{*}}\left(z, \mu_{0}^{2} ; Q^{2}\right),
$$

where the parton-to-virtual photon part is given by a sum of the "QED" contribution and a non-perturbative part:

$$
D_{f \rightarrow \gamma^{*}}\left(z, \mu_{0}^{2} ; Q^{2}\right) \equiv D_{f \rightarrow \gamma^{*}}^{\mathrm{QED}}\left(z, \mu_{0}^{2} ; Q^{2}\right)+D_{f \rightarrow \gamma^{*}}^{\mathrm{NonP}}\left(z, \mu_{0}^{2} ; Q^{2}\right)
$$


$(f=q, \bar{q}, g)$. In the invariant mass cut-off scheme [4], we propose the lowest order QED contribution as

$$
\begin{aligned}
& D_{q \rightarrow \gamma^{*}}^{\mathrm{QED}(0)}\left(z, \mu_{0}^{2} ; Q^{2}\right)=e_{q}^{2}\left(\frac{\alpha_{e m}}{2 \pi}\right)\left[\left(\frac{1+(1-z)^{2}}{z}\right) \ln \left(\frac{\mu_{0}^{2}}{Q^{2} / z+\lambda^{2}}\right)-\left(\frac{Q^{2}}{Q^{2} / z+\lambda^{2}}-\frac{Q^{2}}{\mu_{0}^{2}}\right)\right], \\
& D_{\bar{q} \rightarrow \gamma^{*}}^{\mathrm{QED}(0)}\left(z, \mu_{0}^{2} ; Q^{2}\right)=D_{q \rightarrow \gamma^{*}}^{\mathrm{QED}(0)}\left(z, \mu_{0}^{2} ; Q^{2}\right), \\
& D_{g \rightarrow \gamma^{*}}^{\mathrm{QED}(0)}\left(z, \mu_{0}^{2} ; Q^{2}\right)=0 .
\end{aligned}
$$

In deriving the above lowest-order QED fragmentation functions, we have integrated the invariant mass squared of the fragmenting quark, $k^{2}$, from its lowest value needed to produce a lepton pair of invariant mass $Q$ to the factorization scale $\mu_{0}^{2}$. When $Q$ is small, the lower limit of $k^{2}$ could become sensitive to the current quark mass and to nonperturbative strong interaction dynamics at the scale of $\Lambda_{\mathrm{QCD}}$. We introduce a mass parameter $\lambda$ to prevent the $k^{2}$ integration from entering this strongly interacting regime. If one calculated the lowest-order QED quark-to-virtualphoton fragmentation function with an effective quark mass, $m_{q}$, one would find that $\lambda^{2}=m_{q}^{2} z /(1-z)$ and $z<1$ for a non-vanishing $m_{q}$. However, the value of $\lambda^{2}$ cannot be derived perturbatively. It should be much less than $\mu_{0}^{2} \sim 1 \mathrm{GeV}^{2}$ and should be fixed by experimental data.

When $\lambda^{2}=0$ or $Q^{2} / z \gg \lambda^{2}$, the lowest order QED fragmentation functions in Eq. (13) are reduced to those derived in Ref. 4]. In order to maintain the positivity of the lowest order fragmentation function, and to satisfy the kinematic constraint in Eq. (77), the perturbative input distribution in Eq. (13) is valid for $z \geq Q^{2} /\left(\mu_{0}^{2}-\lambda^{2}\right) \sim Q^{2} / \mu_{0}^{2}$, which is satisfied for the kinematics of interest at RHIC.

The non-perturbative part of the input fragmentation functions in Eq. (12) should cover all other possible contributions including those in Fig. 2(b) and (c) and their potential interferences with each other and that in Fig. 2(a). Similar to what is done in the case of photon structure functions [10] or fragmentation functions [6, 7], we estimate these contributions by adopting the vector meson dominance (VMD) model, for which

$$
\frac{D_{f \rightarrow \gamma}^{\text {NonPert }}\left(z, \mu_{0}^{2} ; Q^{2}\right)}{D_{f \rightarrow V}\left(z, \mu_{0}^{2}\right)} \propto \frac{e^{2}}{f_{V}^{2}}=\frac{4 \pi \alpha_{\mathrm{em}}}{f_{V}^{2}}
$$

with the fragmentation function $D_{f \rightarrow V}$ for a parton of flavor $f$ to a vector meson $V$, and the vector meson decay constant $f_{V}$. Following the Kroll-Wada formula for the Dalitz decay of vector mesons [14], we multiply the fragmentation function in Eq. (14) by a form factor and a phase-space correction factor, to obtain

$$
D_{q \rightarrow \gamma^{*}}^{\mathrm{NonPert}}\left(z, \mu_{0}^{2} ; Q^{2}\right) \propto D_{q \rightarrow V}\left(z, \mu_{0}^{2}\right) \frac{4 \pi \alpha_{\mathrm{em}}}{f_{V}^{2}}\left|F\left(Q^{2}\right)\right|^{2}\left(1-\frac{Q^{2}}{m_{V}^{2}}\right)^{3},
$$

with $F\left(Q^{2}=0\right)=1[14,15]$. In order to take into account any other possible contributions and the potential interference between different production amplitudes, we introduce an unknown constant $\kappa \sim \mathcal{O}(1)$, and choose

$$
D_{q \rightarrow \gamma^{*}}^{\mathrm{NonP}}\left(z, \mu_{0}^{2} ; Q^{2}\right) \equiv \kappa D_{q \rightarrow V}\left(z, \mu_{0}^{2}\right) \frac{4 \pi \alpha_{\mathrm{em}}}{f_{V}^{2}}\left(1-\frac{Q^{2}}{m_{V}^{2}}\right)^{3} .
$$

Here we have absorbed the unknown form factor $F\left(Q^{2}\right)$ into the parameter $\kappa$, in order to minimize the number of free parameters. Although the form factor $F\left(Q^{2}\right)$ depends on $Q^{2}$, we expect a weak $Q^{2}$ dependence when $Q^{2}$ is small [15]. Following [6, 7], we further assume that $m_{V}=m_{\rho}, f_{\rho}^{2} / 4 \pi=2.2$, and $D_{f \rightarrow V} \approx D_{f \rightarrow \pi}$. For our numerical calculations in Sec. IV we thus choose the input fragmentation functions as

$$
D_{f \rightarrow \gamma^{*}}\left(z, \mu_{0}^{2} ; Q^{2}\right)=D_{f \rightarrow \gamma^{*}}^{\mathrm{QED}(0)}\left(z, \mu_{0}^{2} ; Q^{2}\right)+\kappa D_{f \rightarrow \pi}\left(z, \mu_{0}^{2}\right) \frac{4 \pi \alpha_{\mathrm{em}}}{f_{\rho}^{2}}\left(1-\frac{Q^{2}}{m_{\rho}^{2}}\right)^{3} .
$$

We note that future precise experimental data may well require a more refined ansatz, for which one could make use of the details for vector meson form factors and decay rates given in [15].

Note that we have made a "one-photon" approximation throughout, meaning that the lepton pair is always eventually produced by the decay of a single virtual photon. In this approximation, the low-mass dilepton production cross section is equal to the cross section for producing a virtual photon at large transverse momentum, multiplied by a calculable QED factor for the virtual photon to decay into the measured lepton pair, as expressed in Eq. (10). Other than the explicit $Q^{2}$ dependence in the overall QED decay factor, the perturbatively factorized formula in Eq. (10) indicates that any remaining $Q^{2}$ dependence can only come from the short-distance part of the direct contribution, $d \hat{\sigma}_{a b \rightarrow \gamma^{*}(Q) X}^{\operatorname{Dir}} / d Q_{T}^{2} d y$, and from the non-perturbative parton-to-virtual-photon fragmentation functions, $D_{c \rightarrow \gamma^{*}}$. 
Like for the real photon cross section [1], the direct contribution in Eq. (10) is only sensitive to the dynamics at a distance scale $1 / Q_{T} \sim 1 / \mu_{F}$. The logarithmic infrared sensitivity near $Q \gtrsim \Lambda_{\mathrm{QCD}}$ from the Feynman diagrams at next-to-leading order (NLO) or higher orders is canceled between the two terms in Eq. (5) order-by-order in $\alpha_{s}$. The direct contribution is finite when $Q^{2} / Q_{T}^{2} \rightarrow 0$, and its $Q$-dependence could be expanded in terms of a power series in $Q^{2} / Q_{T}^{2} \sim Q^{2} / \mu_{F}^{2}$.

The leading order (LO) direct contribution to virtual photon production is independent of the factorization scheme adopted and becomes the same as the LO direct contribution to the production of a real photon when $Q^{2} / Q_{T}^{2} \rightarrow$ 0 . However, at higher orders in $\alpha_{s}$, the direct contributions to virtual or real photon production depend on the factorization scheme and do not necessarily have to be the same when $Q^{2} / Q_{T}^{2} \rightarrow 0$. For massless prompt photon production, the short-distance hard parts are commonly evaluated by using dimensional regularization and the $\overline{\mathrm{MS}}$ factorization scheme [1]. This means that also collinear singularities in the final state, when a final-state quark radiates the real photon, are subtracted in $\overline{\mathrm{MS}}$. In contrast to this, in our framework for the direct contributions to virtual photon production (see Eq. (5)), we subtract any collinear singularities that arise for $Q^{2} / Q_{T}^{2} \rightarrow 0$ through the asymptotic term, which is a procedure different from $\overline{\mathrm{MS}}$ subtraction. Therefore, starting from NLO, the direct contribution to virtual photon production in the limit $Q^{2} / Q_{T}^{2} \rightarrow 0$ differs from that for real photon production. But, the total production cross section, i.e. the sum of the direct and fragmentation contributions, is not sensitive to the choice of factorization scheme [4, 5].

Partons produced in hard collisions could fragment into virtual photons with either transverse or longitudinal polarization when the photon's invariant mass $Q$ is finite. The fragmentation function for producing longitudinally polarized virtual photons is only significant when the value of the effective momentum fraction $z$ is very small [4, 13]. Because of the steep fall-off of the parton distribution functions as the parton momentum fraction $x$ increases, the virtual photon cross section is dominated by a relatively large $z$ in the fragmentation functions [5], and thus, most large- $Q_{T}$ virtual photons produced in hadronic collisions at RHIC energies should be transversely polarized, like the real photon [16].

As the invariant mass $Q$ of the lepton pair decreases, we expect the virtual photon cross section to become close to the one for producing a real photon, except however for the (approximately) logarithmic $Q^{2}$ dependence of the parton-to-virtual photon fragmentation functions caused by the radiation of a low mass virtual photon which decays into the measured lepton pair. The same QED bremsstrahlung for producing a real photon is absorbed into the parton-to-real-photon fragmentation functions, which of course do not have any explicit $Q^{2}$ dependence and hence no logarithm in the input distribution. On the other hand, the QED channel to produce a lepton pair via a virtual photon, like the one in Fig. 3(a), can be perturbatively calculated, and the potential logarithmic divergence as $Q$ decreases is naturally cut off by the threshold factor $\sqrt{1-4 m_{\ell}^{2} / Q^{2}}$ in Eq. (10) and/or the non-perturbative mass parameter $\lambda^{2}$ in Eq. (13). That is, the logarithmic $Q^{2}$ dependence of parton-to-virtual-photon fragmentation functions from the lowest order QED calculation in Eq. (13) (or, the improved $Q^{2}$ dependence after including higher order contributions) is physical. The $Q^{2}$ dependence is measurable via the invariant mass of the measured lepton pair. We will discuss the consequence of this difference in the $Q^{2}$ dependence between the virtual and real photon cross sections in the next section.

\section{NUMERICAL RESULTS}

In this section, we discuss the phenomenology of low-mass dilepton production in proton-proton as well as nuclear collisions at RHIC. We will mostly consider electron-positron pairs, as relevant for the PHENIX measurements at RHIC [9].

\section{A. Low-mass lepton pairs at large transverse momentum in $p p$ collisions}

We define the invariant cross section for producing lepton pairs in a mass range between $Q_{\min }$ and $Q_{\max }$ as

$$
E \frac{d \sigma_{A B \rightarrow \ell^{+} \ell^{-}(Q) X}}{d^{3} Q} \equiv \int_{Q_{\min }^{2}}^{Q_{\max }^{2}} d Q^{2} \frac{1}{\pi} \frac{d \sigma_{A B \rightarrow \ell^{+} \ell^{-}(Q) X}}{d Q^{2} d Q_{T}^{2} d y}
$$

where the differential dilepton cross section is given in Eq. (10).

The low-mass dilepton cross section vanishes at $Q=2 m_{\ell}$ due to the threshold factor $\sqrt{1-4 m_{\ell}^{2} / Q^{2}}$ in Eq. (10). This value of $Q$ is far lower than the values $Q_{\min }=100 \mathrm{MeV}$ and $Q_{\max }=300 \mathrm{MeV}$ we will consider here. Therefore, among the kinematic factors in Eq. (10) only the term $1 / Q^{2}$ is relevant in the integration over $Q^{2}$. We note that in 
fact for $Q \gg 2 m_{\ell}$ one has $\sqrt{1-4 m_{\ell}^{2} / Q^{2}}\left(1+2 m_{\ell}^{2} / Q^{2}\right)=1+\mathcal{O}\left(m_{\ell}^{4} / Q^{4}\right)$ in (10), amounting to only a tiny correction in the mass region we are considering. Furthermore, as we discussed in the previous section, at $Q \ll Q_{T}$ the factorized perturbative cross section for producing a virtual photon becomes identical to the prompt photon one, except for the approximately logarithmic $Q^{2}$ dependence in the QED parton-to-virtual-photon fragmentation functions. Therefore, we could estimate the invariant cross section in Eq. (18) as follows:

$$
\begin{aligned}
E \frac{d \sigma_{A B \rightarrow \ell^{+} \ell^{-}(Q) X}}{d^{3} Q} & \approx \frac{d \sigma_{A B \rightarrow \gamma(\hat{Q}) X}}{d Q_{T}^{2} d y} \int_{Q_{\min }^{2}}^{Q_{\max }^{2}} d Q^{2}\left(\frac{\alpha_{\mathrm{em}}}{3 \pi^{2} Q^{2}}\right) \sqrt{1-\frac{4 m_{\ell}^{2}}{Q^{2}}}\left(1+\frac{2 m_{\ell}^{2}}{Q^{2}}\right) \\
& \approx \frac{\alpha_{\mathrm{em}}}{3 \pi} \ln \left(\frac{Q_{\max }^{2}}{Q_{\min }^{2}}\right) E_{\gamma} \frac{d \sigma_{A B \rightarrow \gamma(\hat{Q}) X}}{d^{3} Q},
\end{aligned}
$$

where $\hat{Q}^{2}=0$, and $E_{\gamma} d \sigma_{A B \rightarrow \gamma(\hat{Q}) X} / d^{3} Q$ is the prompt photon cross section.

Eq. (19) should be a good approximation if the virtual photon cross section has a very mild $Q^{2}$ dependence and is close to the prompt photon one when $Q_{\min }^{2} \leq Q^{2} \leq Q_{\max }^{2}$. To test this real photon approximation, we introduce an invariant virtual photon cross section,

$$
E \frac{d \sigma_{A B \rightarrow \gamma^{*}(Q) X}}{d^{3} Q}=\frac{1}{\pi} \frac{d \sigma_{A B \rightarrow \gamma^{*}(Q) X}}{d Q_{T}^{2} d y} \approx\left(\frac{3 Q^{2}}{\alpha_{\mathrm{em}}}\right) \frac{d \sigma_{A B \rightarrow \ell^{+} \ell^{-}(Q) X}}{d Q^{2} d Q_{T}^{2} d y},
$$

where the dilepton differential cross section is given in Eq. (10) and terms of order $m_{\ell}^{2} / Q^{2}$ have been neglected.

In order to generate the numerical values for the dilepton cross section in the figures below, we let the fragmentation scale, $\mu_{F}$, be equal to the renormalization scale, $\mu$, and choose $\mu_{F}=\mu=\sqrt{Q^{2}+Q_{T}^{2}}$. We use the NLO CTEQ6M parton distributions for the unpolarized nucleon [17]. We solve for the parton-to-virtual photon fragmentation functions by using the inhomogeneous evolution equation in Eq. (6) and the input fragmentation functions in Eq. (17) at $\mu_{0}=1 \mathrm{GeV}$ and $\lambda=200 \mathrm{MeV}$. For the parton-to-pion fragmentation functions needed for the non-perturbative input fragmentation functions in Eq. (17), we use the parameterization derived in Ref. [18].
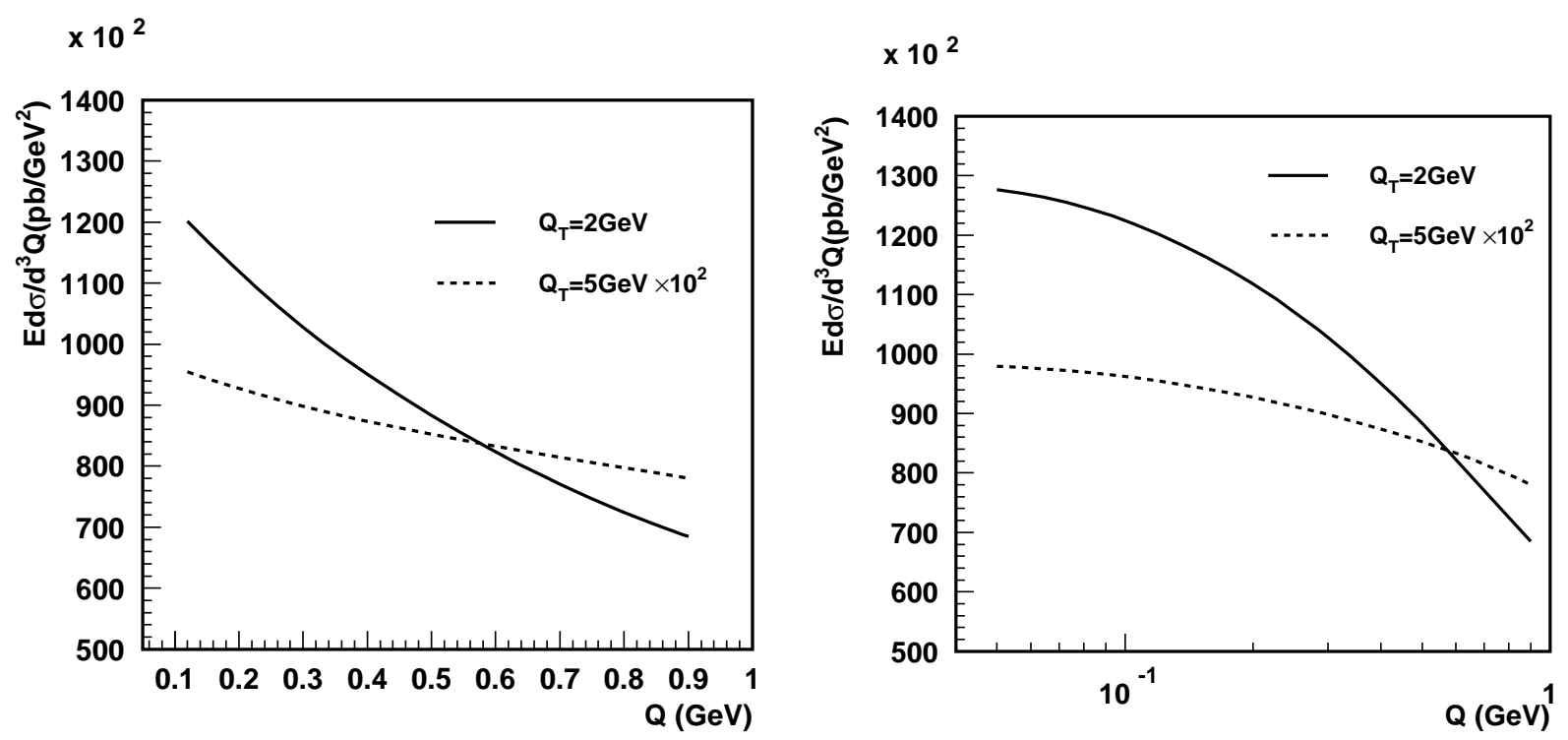

FIG. 4: Invariant virtual photon cross section in Eq. (20) as a function of the photon's invariant mass $Q$ in proton-proton collision at $\sqrt{s}=200 \mathrm{GeV}, y=0$, and $Q_{T}=2,5 \mathrm{GeV}$. Note that the results for $Q_{T}=5 \mathrm{GeV}$ have been multiplied by a factor 100. The plot on the right-hand-side has a logarithmic scale of the abscissa, in order to better illustrate the small- $Q$ region.

In Fig. 4. we plot the invariant virtual photon cross section as a function of $Q$ in proton-proton collisions at $\sqrt{s}=200 \mathrm{GeV}$ and rapidity $y=0$. The solid line is for $Q_{T}=2 \mathrm{GeV}$, while the dashed one is for $Q_{T}=5 \mathrm{GeV}$. From the figure on the left it is clear that the $Q^{2}$ dependence of the virtual photon cross section is relatively weak for $Q_{T}=5 \mathrm{GeV}$, while it becomes more significant when $Q_{T}$ is small. Therefore, for the range of $Q$ of interest for PHENIX [9], the real photon approximation in Eq. (19) should be reasonable if $Q_{T}>5 \mathrm{GeV}$, while it will become less accurate below that. In the figure on the right, we plot the cross section for the $Q$ as small as $50 \mathrm{MeV}$. The logarithmic plot clearly 


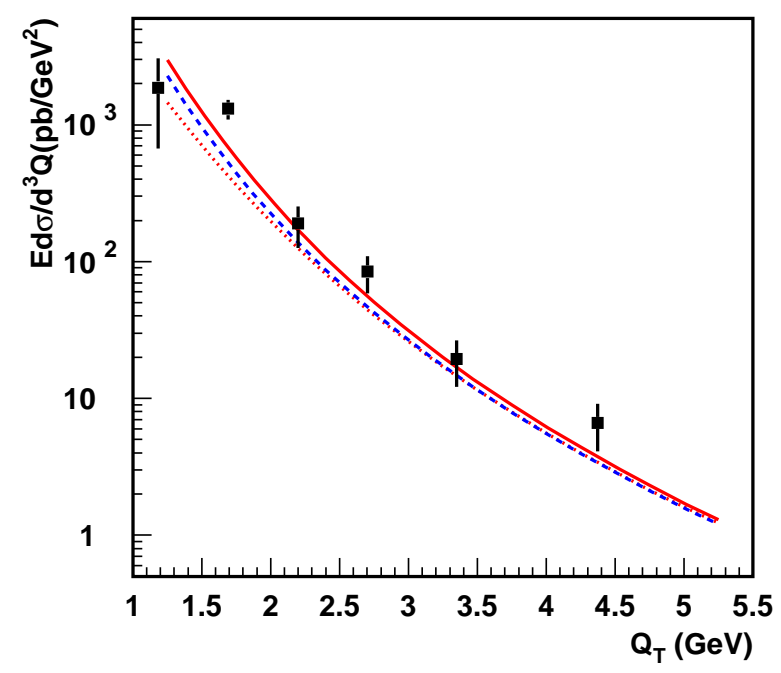

FIG. 5: Invariant cross section for low-mass dilepton production, defined in Eq. (18), as a function of the pair's transverse momentum in proton-proton collisions at $\sqrt{s}=200 \mathrm{GeV}$ and $y=0$, for $\kappa=1$ (solid line) and for $\kappa=0$ (dotted line). Also shown is the approximate cross section evaluated by using Eq. (19) with a NLO prompt photon cross section (dashed). The data are from Ref. [9].

shows the slowing down of the growth of the cross section when $Q \lesssim \lambda=200 \mathrm{MeV}$. The virtual photon cross section should asymptotically approach the real photon cross section as $Q \rightarrow 0$.

In Fig. [5. we show the invariant cross section for producing low mass electron-positron pairs, defined in Eq. (18), as a function of the pair's transverse momentum, $Q_{T}$, in proton-proton collisions at $\sqrt{s}=200 \mathrm{GeV}$ and rapidity $y=0$. We have included the NLO hard part for the direct contribution [5, 19] and the LO hard part for the fragmentation contribution, which matches our choice of a LO fragmentation function 20]. As mentioned earlier, we choose $Q_{\min }=100 \mathrm{MeV}$ and $Q_{\max }=300 \mathrm{MeV}$, to match the kinematics of the PHENIX measurements [9], and $\lambda=200 \mathrm{MeV}$. We find that setting the non-perturbative mass parameter $\lambda$ to zero increases the total cross section by a few percent. To test the uncertainty associated with the non-perturbative input fragmentation functions, we plot the cross section under two assumptions: $\kappa=1$ (solid line) and $\kappa=0$ (dotted line). The choice $\kappa=0$ corresponds to fragmentation functions generated without any hadronic input, which should be considered as the lower limit for our numerical predictions. On the other hand, $\kappa=1$ corresponds to an almost maximal hadronic contribution, since it amounts to neglecting all potential interferences between the different sources of lepton production and to setting the form factor to unity. We thus expect that the range between the solid and the dotted line should roughly represent the uncertainty of our prediction due to the use of our model for the input fragmentation function, up to a few percent additional uncertainty related to the choice of $\lambda^{2}$. For comparison, we also show in Fig. 5 the approximate invariant dilepton cross section in Eq. (19), using a full NLO prompt photon cross section (dashed line) [20] with the parton-to-photon fragmentation functions of [6]. One can see that the latter result has the similar $Q_{T}$ shape as the one for the choice $\kappa=1$ in the full calculation, but a smaller overall normalization.

As we mentioned earlier, the non-perturbative parton-to-virtual-photon fragmentation functions have not been measured independently, and the normalization of the low-mass dilepton cross section calculated here is sensitive to the relative size of the fragmentation contribution. We find that the fragmentation contribution is a fairly important part of the production rate. It is dominated by the QED logarithmic term in the input distribution in our model, see Eq. (13). This logarithmic $Q^{2}$ dependence is one origin of the difference in the production rates found for virtual and real photons in Fig. 5. The approximate invariant dilepton cross section in Eq. (19), which is based on the full NLO prompt photon cross section, neglects any $Q^{2}$ dependence of the fragmentation contribution of the full calculation, which is not very small for the range of $Q$ relevant to the PHENIX data as indicated in Fig. 目. The logarithmic $Q^{2}$ dependence of the fragmentation functions, in combination with the factor $1 / Q^{2}$ in Eq. (11), could generate a term proportional to a double logarithm $\ln ^{2}\left(Q_{\max }^{2} / Q_{\min }^{2}\right)$ and enhance the overall production rate in comparison to the single logarithm $\ln \left(Q_{\max }^{2} / Q_{\min }^{2}\right)$ in Eq. (19). Also, our full calculation uses only the LO hard part and LO partonto-virtual-photon fragmentation functions. Using a NLO hard part and NLO parton-to-virtual-photon fragmentation functions, which are not available yet, would be expected to further increase our predicted production rate. In any

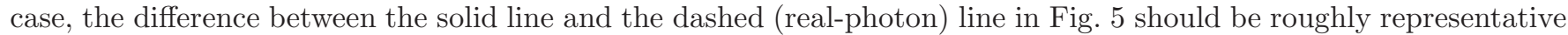
of the present theoretical uncertainty of the calculation of the low-mass dilepton cross section for the given kinematics. 

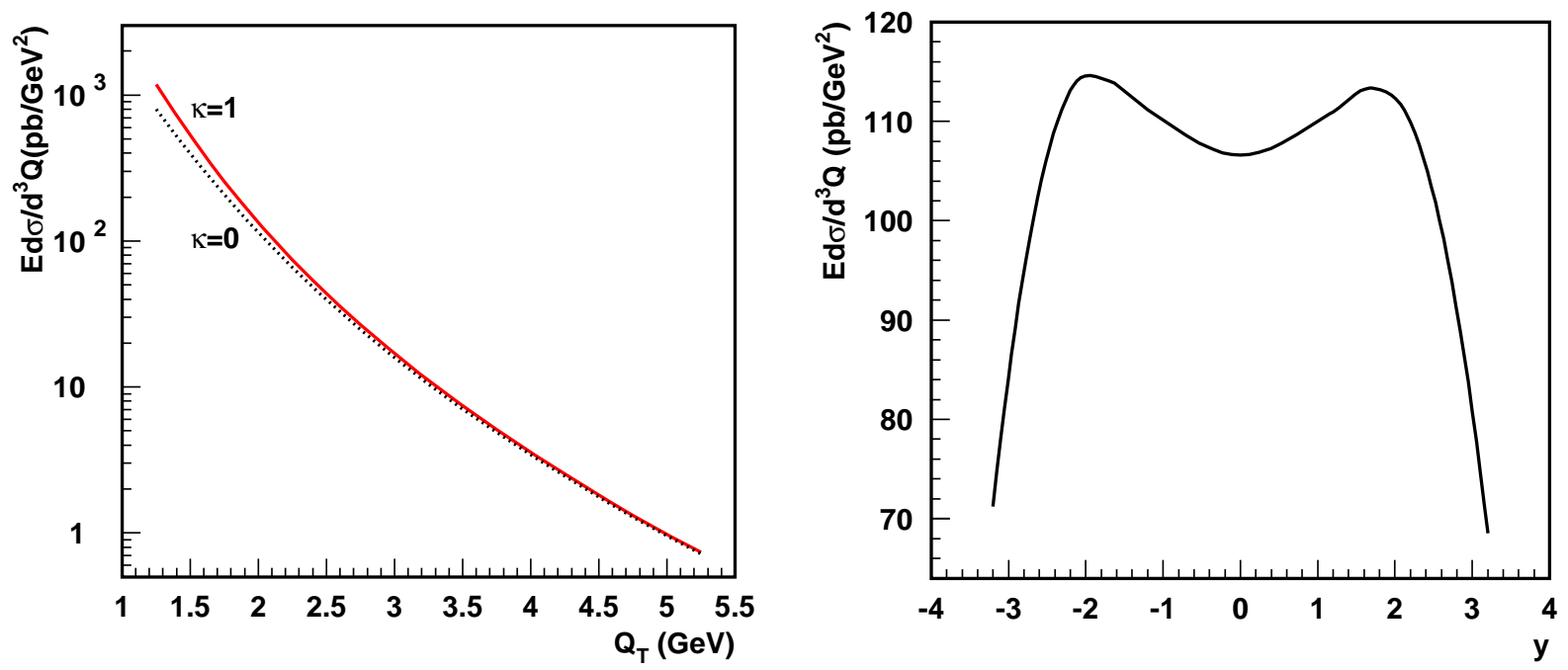

FIG. 6: Invariant cross section for low mass $\mu^{+} \mu^{-}$production as a function of the transverse momentum $Q_{T}$ at $y=1.7$, and as a function of the rapidity at $Q_{T}=2 \mathrm{GeV}$. The solid line is for $\kappa=1$ and the dotted line is for $\kappa=0$.

In Fig. 6. we plot the invariant cross section for low-mass dimuon production in proton-proton collisions at $\sqrt{s}=$ $200 \mathrm{GeV}$. We choose here the range $Q_{\min }=250 \mathrm{MeV}$ to $Q_{\max }=550 \mathrm{MeV}$, so that we avoid both the mass threshold at $Q=2 m_{\mu} \approx 211 \mathrm{MeV}$ and the $\rho$-meson resonance. We plot the invariant cross section as a function of the dimuon's transverse momentum $Q_{T}$ at a forward rapidity $y=1.7$, which is roughly the average rapidity covered by the PHENIX muon arm. We again present two curves: the solid line corresponds to $\kappa=1$ and the dotted one to $\kappa=0$. In the right part of the figure, we show the invariant cross section as a function of $y$ at $Q_{T}=2 \mathrm{GeV}$ and $\kappa=0$. Because of the larger invariant mass and more forward angles we are considering here, the production rate is smaller than in the electron-positron case.

\section{B. Isospin effects in nuclear low-mass dilepton production}

Like prompt photon production, the hadronic production of low-mass lepton pairs is also dominated by the $q g \rightarrow \gamma^{*} q$ Compton subprocess. Therefore, the invariant cross section for low-mass dilepton production could have significant isospin effects in nuclear collisions, similar to the ones observed in recent studies of the real photon cross section [21, 22].

In order to present nuclear effects in high energy nuclear collisions, one often defines a nuclear modification factor as the ratio of the nuclear cross section, normalized to the number of binary collisions, over the proton-proton one. As the proton-proton cross section differs from that for proton-neutron or neutron-neutron scattering, the nuclear modification factor will be different from unity simply by isospin effects, even in the absence of any "genuine" nuclear effects. To quantify the size of the isospin effect in deuteron-gold collisions, we introduce the ratio

$$
R_{\mathrm{dAu}}^{\mathrm{iso}} \equiv \frac{\frac{1}{2 A} d^{2} \sigma^{\mathrm{dAu}} / d Q_{T} d y}{d^{2} \sigma^{p p} / d Q_{T} d y}
$$

where the deuteron-gold cross section in the numerator is calculated by replacing the proton's parton distribution functions (PDFs) by

$$
f_{i}^{p}\left(x, Q^{2}\right) \rightarrow\left[Z \cdot f_{i}^{p}+(A-Z) \cdot f_{i}^{n}\right] / A,
$$

in the $p p$ calculation, with $A=197(A=2)$ and $Z=79(Z=1)$ the atomic weight and proton number of the gold (deuteron) nucleus, respectively. For gold-gold collisions, we introduce a corresponding $R_{\mathrm{AuAu}}^{\mathrm{iso}}$.

In Fig. 7 we plot $R_{\mathrm{dAu}}^{\text {iso }}$ and $R_{\mathrm{AuAu}}^{\text {iso }}$ for low-mass electron-positron pair production as functions of $Q_{T}$. As before, we integrate over the mass range $100 \leq Q \leq 300 \mathrm{MeV}$. The solid lines are for $\kappa=1$ while the dotted ones are for $\kappa=0$. We find that the isospin effect is significant, giving rise to a reduction of the nuclear modification factor by as much as $10 \%$ at $Q_{T} \sim 10 \mathrm{GeV}$. This finding is consistent with the results obtained for real photon production in 21, 22]. This reduction is readily understood: for low-mass dilepton production at large transverse momentum, the $q g \rightarrow \gamma^{*} q$ Compton subprocess dominates the production rate. In the case of proton-proton collisions, the Compton 

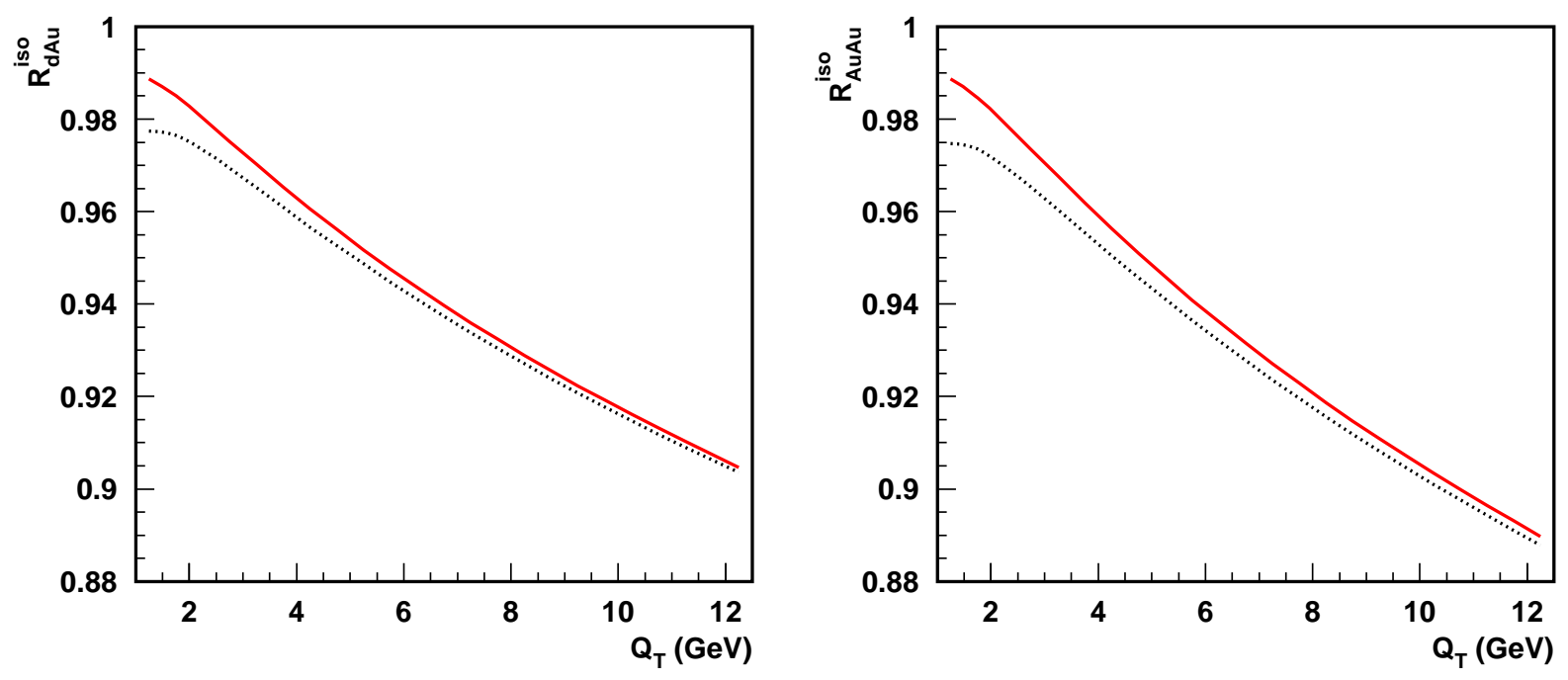

FIG. 7: $R_{\mathrm{dAu}}^{\text {iso }}$ and $R_{\mathrm{AuAu}}^{\text {iso }}$ as functions of $Q_{T}$ at $\sqrt{s}=200 \mathrm{GeV}$ and $y=0: \kappa=1$ (solid), $\kappa=0$ (dotted).

process involves the quark combination $\frac{4}{9} f_{u}^{p}+\frac{1}{9} f_{d}^{p}$. On the other hand, for the case of neutron-neutron collisions, this combination becomes $\frac{4}{9} f_{u}^{n}+\frac{1}{9} f_{d}^{n}=\frac{4}{9} f_{d}^{p}+\frac{1}{9} f_{u}^{p}$. Since $f_{u}^{p}>f_{d}^{p}$, the invariant cross section at central rapidities follows the relation: $\sigma^{n n}<\sigma^{n p}=\sigma^{p n}<\sigma^{p p}$. As a result, $R_{A B}^{\text {iso }}<1$ for any nucleus-nucleus collision. In addition, the stronger suppression with increasing $Q_{T}$ is caused by the fact that the ratio $f_{d}^{p}(x) / f_{u}^{p}(x)$ decreases as $x$ increases.

The dependence of the results on $\kappa$ in Fig. [7 arises in the following way. When $\kappa=1$, the fragmentation contribution is much larger. Since this contribution has a substantial contribution from the gluon-gluon fusion subprocess, it is much less sensitive to any isospin effect. Consequently, for the $\kappa=1$ case one finds a smaller isospin suppression at low $Q_{T}$, whereas at higher $Q_{T}$, where the fragmentation contribution becomes overall less important, the results become independent of $\kappa$.

\section{Low-mass lepton pairs in nuclear collisions}

Since the cross section for low-mass lepton pairs at large transverse momentum is dominated by the $q g$ Compton subprocesses, it is expected to be an excellent probe of the gluon distribution function [3, 5], and its nuclear dependence. In this subsection, we investigate this issue by studying the dependence of the cross section on the choice of nuclear parton distribution functions (nPDFs). We neglect any other nuclear effects, such as saturation effects 23], parton multiple scattering in the nuclear medium [22, 24, 25], or thermal radiation of lepton pairs [26]. The nPDFs, or more precisely, the effective PDFs of a bound proton inside a nucleus of atomic weight $A$ are often written as

$$
f_{i}^{p / A}\left(x, Q^{2}\right) \equiv \rho_{i}^{A}\left(x, Q^{2}\right) f_{i}^{p}\left(x, Q^{2}\right)
$$

where the factors $\rho_{i}^{A}\left(x, Q^{2}\right)$ are usually determined in global QCD fits to the data from deep inelastic lepton scattering off nuclei [27, 28, 29, 30, 31]. The nuclear PDFs for a bound neutron are obtained from those for the proton by using isospin symmetry. For example, $f_{u}^{n / A}=f_{d}^{p / A}=\rho_{d}^{A}\left(x, Q^{2}\right) f_{d}^{p}$. Following Eq. (22) we then obtain the parton distribution for flavor $i$ in the nucleus as

$$
\left[Z \cdot f_{i}^{p / A}+(A-Z) \cdot f_{i}^{n / A}\right] / A
$$

The nuclear modification factor for deuteron-nucleus collisions is defined as

$$
R_{\mathrm{dAu}} \equiv \frac{1}{\left\langle N_{\text {coll }}\right\rangle} \frac{d^{2} N^{\mathrm{dAu}} / d Q_{T} d y}{d^{2} N^{p p} / d Q_{T} d y} \stackrel{\text { min.bias }}{=} \frac{\frac{1}{2 A} d^{2} \sigma^{\mathrm{dAu}} / d Q_{T} d y}{d^{2} \sigma^{p p} / d Q_{T} d y},
$$

where $\left\langle N_{\text {coll }}\right\rangle$ is the number of binary collisions and the label "min. bias" refers to minimum bias events. The modification factor for gold-gold collisions, $R_{\mathrm{AuAu}}$, is defined analogously.

In order to investigate the sensitivity of the nuclear modification factors to the nuclear gluon distribution, we use three sets of nPDFs in our plots below: EKS98 [27], dFS2003 [30], and the recent EPS08 set [31]. We emphasize that 


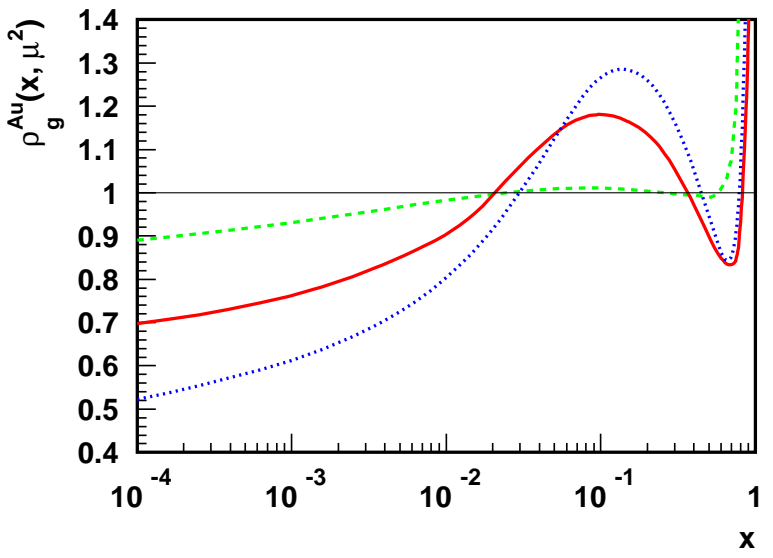

FIG. 8: The ratio $\rho_{g}^{\mathrm{Au}}\left(x, \mu^{2}\right)$ of the gluon distributions in a gold nucleus and in the proton, defined in Eq. (23), as a function of $x$ at $\mu=3 \mathrm{GeV}$, evaluated for three sets of nPDFs: EKS98 (solid), EPS08 (dotted), and dFS2003 (dashed).

none of the three sets considers the dependence of the nPDFs on the transverse location inside the nucleus (the impact parameter dependence) [32]. Using Eq. (23) implies that we average over the impact parameter dependence of the nuclear effects. While all sets fit the data from which the nPDFs were extracted, they predict a significantly different gluon distribution function in a large nucleus, as shown in Fig. 8. While the gluon distribution of dFS2003 has an overall relatively weak nuclear dependence, the other two sets have strong shadowing at small $x$ and anti-shadowing at $x \sim 0.1$, in particular for EPS08.
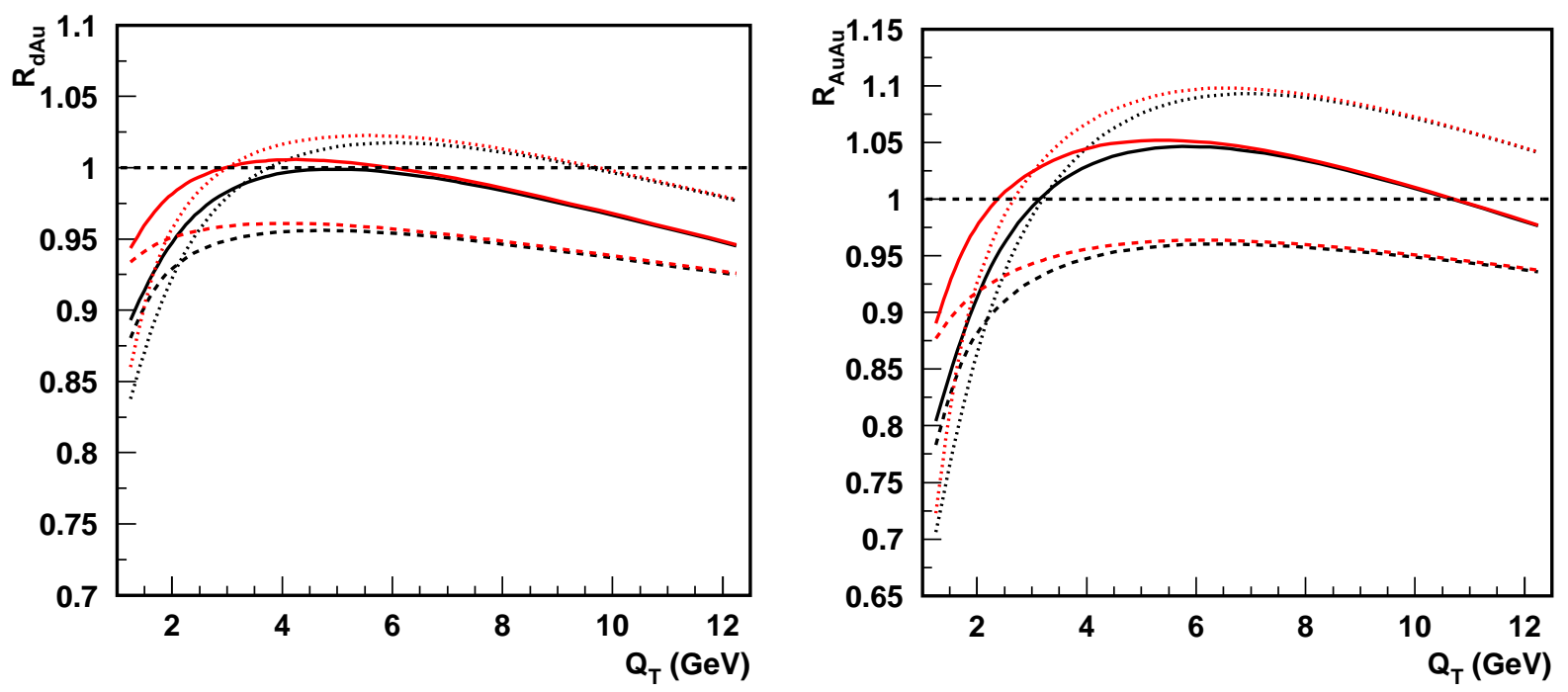

FIG. 9: The nuclear modification factor for the invariant cross section for low-mass dilepton production, $R_{\mathrm{dAu}}$ and $R_{\mathrm{AuAu}}$, as functions of $Q_{T}$ at $\sqrt{s}=200 \mathrm{GeV}$ and $y=0$. The solid, dashed, and dotted lines correspond to the EKS98, dFS2003, and EPS08 sets of nPDFs, respectively. For each set, the top curve (red color online) is for $\kappa=1$ and the bottom one (black color online) for $\kappa=0$.

In Fig. 9 we plot the nuclear modification factors $R_{\mathrm{dAu}}$ and $R_{\mathrm{AuAu}}$ for the invariant cross section for low-mass electron-positron pair production in nuclear collisions as functions of the lepton pair's transverse momentum, at $\sqrt{s}=200 \mathrm{GeV}$ and $y=0$. As expected from the differences in the nuclear gluon distribution shown in Fig. 8 the three sets of nPDFs lead to significant differences in the predictions for the nuclear modification factors. One can first of all see that the EPS08 set gives modification factors that become larger than unity at $Q_{T} \gtrsim 3 \mathrm{GeV}$. This implies that for the kinematics we are considering the anti-shadowing region dominates, which as we saw is most pronounced for the EPS08 set. The EKS98 set with its slightly lower anti-shadowing still leads to $R_{\mathrm{AuAu}}>1$ at $Q_{T} \gtrsim 3 \mathrm{GeV}$, while for dFS2003, which hardly has any anti-shadowing of the gluon distribution at all, both $R_{\mathrm{dAu}}$ and $R_{\mathrm{AuAu}}$ remain below unity. Since at mid-rapidity the momentum fractions of the two colliding partons are on average the same, it is not surprising that the anti-shadowing contributions are amplified in $R_{\mathrm{AuAu}}$, as shown by $R_{\mathrm{AuAu}}>R_{\mathrm{dAu}}$ at high 

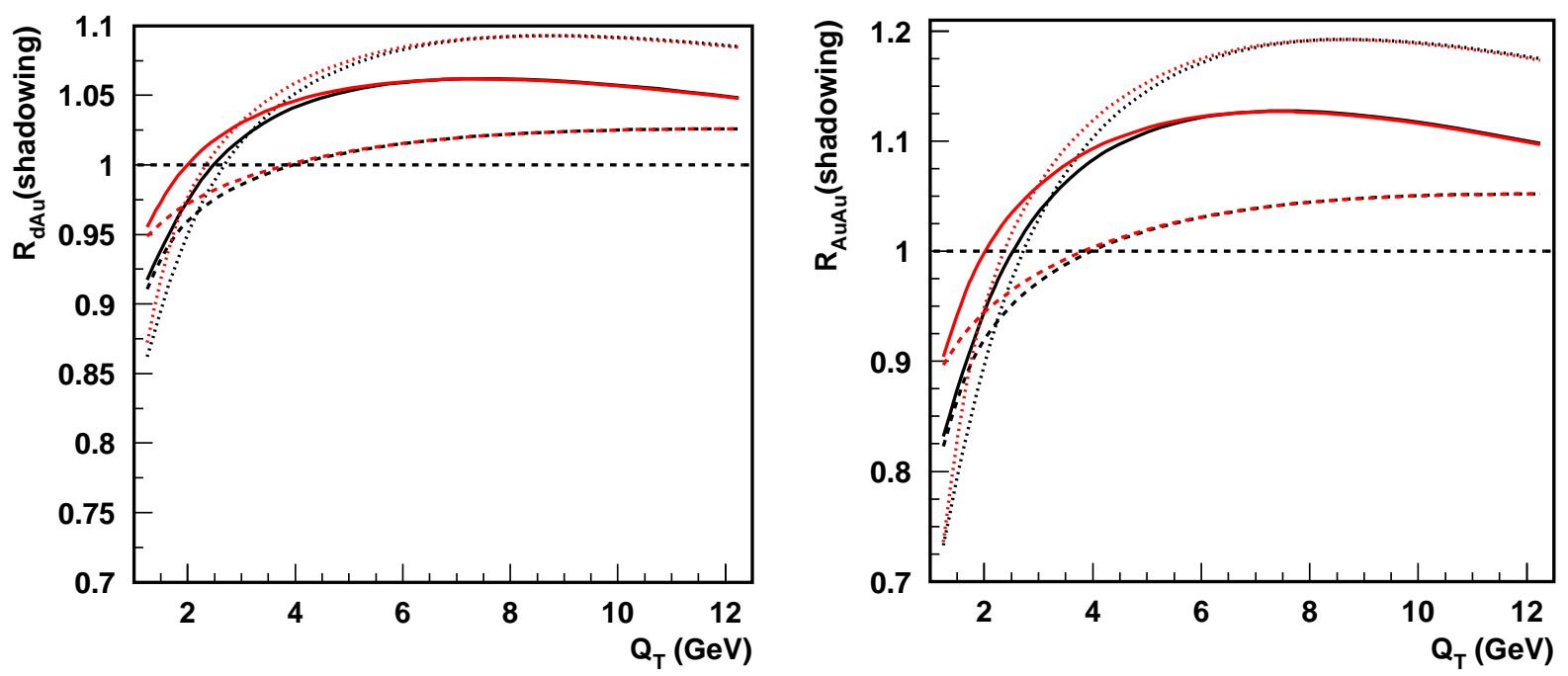

FIG. 10: Same as in Fig. 9 but ignoring the isospin effects of the nuclear PDFs. These plots thus represent the nuclear shadowing and anti-shadowing effects in isolation.

$Q_{T}$. We thus conclude that the nuclear modification factor for the production of low-mass electron-positron pairs at RHIC may provide valuable information on the nuclear gluon distribution. We note that of course the nuclear effects in the quark (and anti-quark) distributions also matter for the precise behavior of $R_{\mathrm{dAu}}$ and $R_{\mathrm{AuAu}}$; however, these distributions are much better known than the gluon one and are more similar in the various nPDF sets.

For all three sets of nPDFs we consider, $R_{\mathrm{dAu}}$ and $R_{\mathrm{AuAu}}$ decrease at very high $Q_{T}$ and tend to go below unity. The nuclear modification factors evaluated using the dFS2003 set actually remain below unity in the entire range of $Q_{T}$. This turns out to be a result of the isospin effect we discussed earlier, which pushes the nuclear modification factors to lower values. In order to verify this, we plot in Fig. 10 the same nuclear modification factors, evaluated however ignoring the isospin effect by setting $A=Z$ in Eq. (24). As expected, the nuclear modification factor in Fig. 10 then follows the features of the nuclear gluon distribution more closely.

We note that some of the features we find have also been observed in studies of the real photon cross section [21, 22]. We remind the reader that the importance of the low-mass dilepton observable lies in the fact that it allows to extend the measurements to lower $Q_{T} \lesssim 5 \mathrm{GeV}$. For real prompt photons, preliminary PHENIX measurements find $R_{\mathrm{dAu}}$ [33] and $R_{\mathrm{AuAu}}$ [34] to be consistent with unity within relatively large errors, with $R_{\mathrm{AuAu}}$ appearing to drop to lower values at $Q_{T} \gtrsim 12 \mathrm{GeV}$.

Finally, we close this section by showing in Fig. 11 a comparison of our calculated low-mass dilepton cross section in $\mathrm{Au}+\mathrm{Au}$ collisions with the recent RHIC data at $\sqrt{s}=200 \mathrm{GeV}$ and $y=0$ [9]. We use Eq. (18), the EPS08 nPDFs, and $\kappa=1$ (solid line), $\kappa=0$ (dotted line). We take into account the centrality dependence of nuclear collisions by scaling the calculated cross section with the average Glauber nuclear overlap function $\left\langle T_{A A}\right\rangle$, which is the same as that used by the PHENIX experiments [35]. We find that thanks to the anti-shadowing effect the calculated cross section is roughly consistent with the experimental data for $Q_{T}>3 \mathrm{GeV}$. Without including other potential nuclear effects, such as power corrections from parton multiple scattering [24] or thermal radiation [26], which could enhance the dilepton cross section at low $Q_{T}$, our calculated cross section in $\mathrm{Au}+\mathrm{Au}$ collisions with the nuclear modification from nPDFs alone cannot explain the existing RHIC data. As shown by Fig. 11, the production rate of low-mass lepton pairs in $\mathrm{Au}+\mathrm{Au}$ collisions measured by PHENIX is clearly larger than the perturbative QCD calculation for $Q_{T} \lesssim 3 \mathrm{GeV}$. As pointed out in Ref. [21], energy loss of partons in the fragmentation contribution could generate additional nuclear dependence of the direct photon cross section, which could reduce the production rate in $\mathrm{Au}+\mathrm{Au}$ collisions. We expect the effect of energy loss of the fragmentating partons on low mass dilepton production to be similar.

\section{SUMMARY}

We have investigated the production cross section for lepton pairs in the regime $Q_{T} \gg Q \sim \Lambda_{\mathrm{QCD}}$. We have argued that, like for real photons, the cross section can be systematically factorized into universal fragmentation functions, parton distributions, and perturbatively calculable partonic hard parts evaluated at a distance scale $\sim \mathcal{O}\left(1 / Q_{T}\right)$. We conclude that the lepton-pair cross section in this kinematic regime can be treated in a similar fashion, and with 

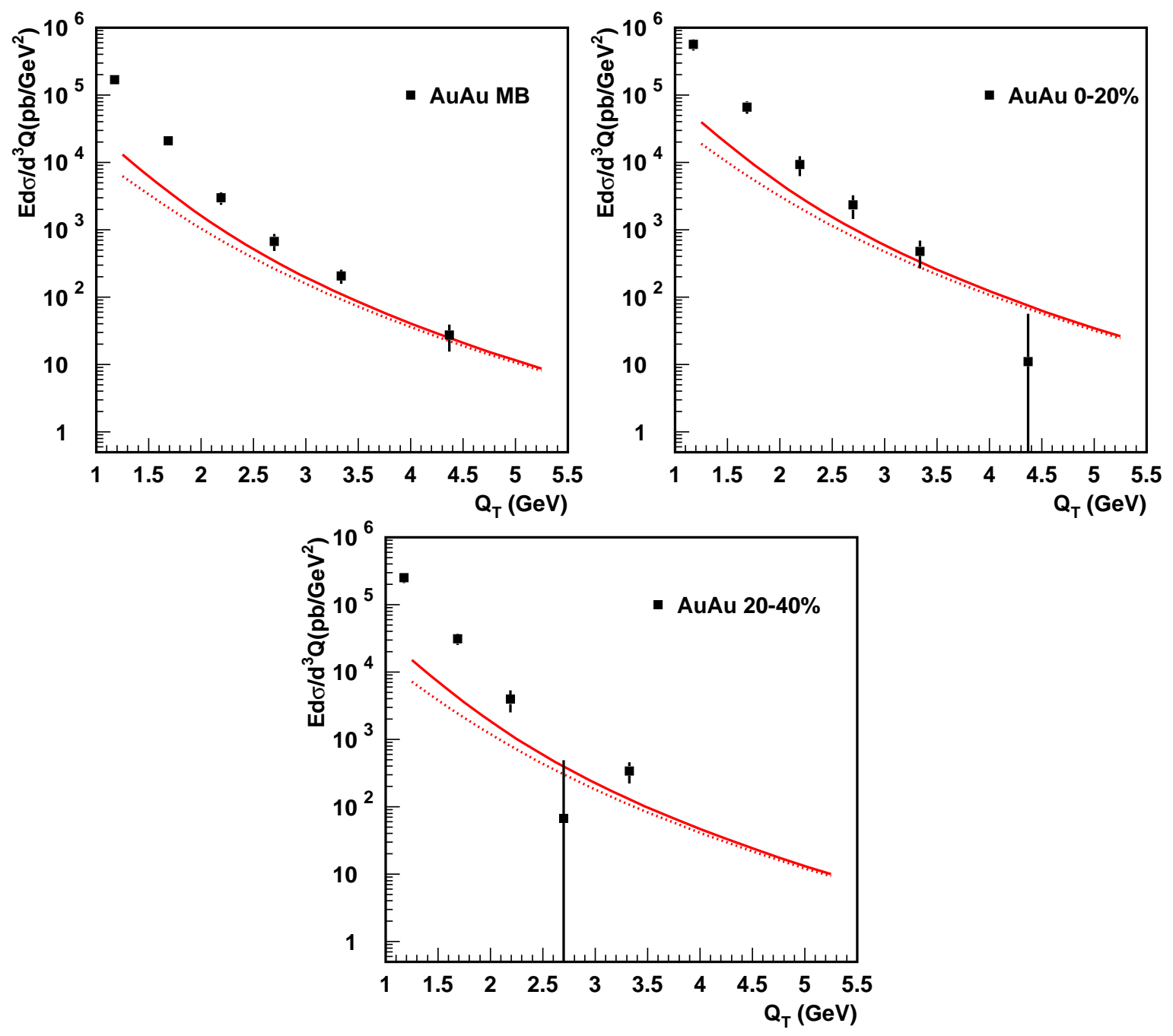

FIG. 11: Invariant cross section for low-mass dilepton production in Au+Au collisions as a function of the pair's transverse momentum, evaluated by using Eq. (18) and the EPS0 8 nPDFs at $\sqrt{s}=200 \mathrm{GeV}$ and $y=0$. We use $\kappa=1$ (solid) and $\kappa=0$ (dotted). The centrality dependence of nuclear collisions is taken into account by scaling the cross section with the Glauber nuclear overlap function [35]. Experimental data are from Ref. [9].

similar rigor, as the real-photon one.

When $Q \sim \Lambda_{\mathrm{QCD}}$, the fragmentation contribution to the lepton-pair cross section cannot be completely perturbative as it is for $Q \gg \Lambda_{\mathrm{QCD}}$. We have introduced models for the lepton pair input fragmentation functions at a scale $\mu_{0} \sim 1 \mathrm{GeV}$, which we have evolved perturbatively to higher scales. Using the evolved fragmentation functions, we have calculated the transverse momentum distributions of low-mass lepton pairs in proton-proton collisions at $\sqrt{s}=200 \mathrm{GeV}$ at RHIC. Our calculated cross section is consistent with existing RHIC data 9 ]. A careful comparison with the existing and future data should help to better pin down the universal fragmentation functions.

We have also discussed the case of nuclear collisions at RHIC, and have found that the nuclear modification factors in deuteron-gold and gold-gold collisions for high- $Q_{T}$ low-mass dilepton production are good probes of the nuclear gluon distribution, similar to those for real photon production. We also noticed that isospin effects are very significant for the nuclear modification factors. We demonstrated that our calculated cross section in $\mathrm{Au}+\mathrm{Au}$ collisions with the nuclear modification from nPDFs alone cannot explain the existing RHIC data. The data from $\mathrm{Au}+\mathrm{Au}$ collisions show a clear enhancement over the perturbative QCD calculation for $Q_{T} \lesssim 3 \mathrm{GeV}$.

We finally stress that the production of low-mass lepton pairs and the prompt photons at large transverse momentum in high energy hadronic collisions have many features in common. However, experimentally, they are also complementary to each other, since they can be exploited in distinct kinematic regimes. A combination of these two electromagnetic probes of hard scattering could provide good information on gluon distributions and short-distance QCD dynamics. 


\section{Acknowledgments}

We thank Y. Akiba for many useful discussions on hadronic production of low-mass lepton pairs, and for his careful reading and valuable comments on our manuscript. We are grateful to E.L. Berger for discussions on DrellYan production of low-mass lepton pairs and to M. Strikman and R. Venugopalan for discussions on nuclear parton distributions. This work was supported in part by the U.S. Department of Energy under grant number DE-FG0287ER40371 (JQ) and contract number DE-AC02-98CH10886 (WV). JQ thanks the Institute of High Energy Physics, Chinese Academy of Science for its hospitality during the writing of this work.

[1] for reviews, see: J. F. Owens, Rev. Mod. Phys. 59, 465 (1987); W. Vogelsang and M. R. Whalley, J. Phys. G 23, A1 (1997).

[2] P. Aurenche, R. Baier and M. Fontannaz, Phys. Lett. B 209, 375 (1988).

[3] E. L. Berger, L. E. Gordon and M. Klasen, Phys. Rev. D 58, 074012 (1998) arXiv:hep-ph/9803387.

[4] J. W. Qiu and X. f. Zhang, Phys. Rev. D 64, 074007 (2001) arXiv:hep-ph/0101004.

[5] E. L. Berger, J. W. Qiu and X. f. Zhang, Phys. Rev. D 65, 034006 (2002) arXiv:hep-ph/0107309].

[6] M. Glück, E. Reya and A. Vogt, Phys. Rev. D 48, 116 (1993) [Erratum-ibid. D 51, 1427 (1995)].

[7] L. Bourhis, M. Fontannaz and J. P. Guillet, Eur. Phys. J. C 2, 529 (1998) arXiv:hep-ph/9704447.

[8] A. Gehrmann-De Ridder and E. W. N. Glover, Nucl. Phys. B 517, 269 (1998) arXiv:hep-ph/9707224.

[9] A. Adare et al. [PHENIX Collaboration], arXiv:0804.4168 [nucl-ex].

[10] T. Uematsu and T. F. Walsh, Phys. Lett. B 101, 263 (1981); Nucl. Phys. B 199, 93 (1982); W. Ibes and T. F. Walsh, Phys. Lett. B 251, 450 (1990); G. Rossi, Phys. Rev. D 29, 852 (1984); F. Borzumati and G. A. Schuler, Z. Phys. C 58, 139 (1993); M. Glück, E. Reya and M. Stratmann, Phys. Rev. D 51, 3220 (1995); Phys. Rev. D 54, 5515 (1996) arXiv:hep-ph/9605297; G. A. Schuler and T. Sjostrand, Z. Phys. C 68, 607 (1995) arXiv:hep-ph/9503384; Phys. Lett. B 376, 193 (1996) arXiv:hep-ph/9601282; M. Drees and R. M. Godbole, Phys. Rev. D 50, 3124 (1994) arXiv:hep-ph/9403229; D. de Florian, C. Garcia Canal and R. Sassot, Z. Phys. C 75, 265 (1997) arXiv:hep-ph/9608438; M. Klasen, G. Kramer and B. Pötter, Eur. Phys. J. C 1, 261 (1998) arXiv:hep-ph/9703302; G. Kramer and B. Pötter, Eur. Phys. J. C 5, 665 (1998) arXiv:hep-ph/9804352]; M. Glück, E. Reya and I. Schienbein, Phys. Rev. D 60, 054019 (1999) [Erratum-ibid. D 62, 019902 (2000)] arXiv:hep-ph/9903337]; Phys. Rev. D 63, 074008 (2001) arXiv:hep-ph/0009348.

[11] P. J. Bussey [H1 Collaboration and ZEUS Collaboration], Nucl. Phys. Proc. Suppl. 126, 17 (2004) arXiv:hep-ex/0307071, and references therein.

[12] J. C. Collins, D. E. Soper and G. Sterman, Adv. Ser. Direct. High Energy Phys. 5, 1 (1988) arXiv:hep-ph/0409313.

[13] E. Braaten and J. Lee, Phys. Rev. D 65, 034005 (2002) arXiv:hep-ph/0102130.

[14] N. M. Kroll and W. Wada, Phys. Rev. 98, 1355 (1955).

[15] A. Fäßler, C. Fuchs and M. I. Krivoruchenko, Phys. Rev. C 61, 035206 (2000) arXiv:nucl-th/9904024.

[16] J. W. Qiu, R. Rodriguez and X. f. Zhang, Phys. Lett. B 506, 254 (2001) arXiv:hep-ph/0102198.

[17] J. Pumplin, D. R. Stump, J. Huston, H. L. Lai, P. Nadolsky and W. K. Tung, JHEP 0207, 012 (2002) arXiv:hep-ph/0201195.

[18] B. A. Kniehl, G. Kramer and B. Pötter, Nucl. Phys. B 582, 514 (2000) arXiv:hep-ph/0010289.

[19] E. L. Berger, L. E. Gordon and M. Klasen, Phys. Rev. D 58, 074012 (1998) arXiv:hep-ph/9803387].

[20] here we have used the Fortran code of: L. E. Gordon and W. Vogelsang, Phys. Rev. D 48, 3136 (1993); Phys. Rev. D 50, 1901 (1994).

[21] F. Arleo, JHEP 0609, 015 (2006) arXiv:hep-ph/0601075.

[22] S. Turbide, C. Gale, E. Frodermann and U. Heinz, Phys. Rev. C 77, 024909 (2008) arXiv:0712.0732 [hep-ph]]; I. Vitev and B. W. Zhang, Phys. Lett. B 669, 337 (2008) arXiv:0804.3805 [hep-ph]]; arXiv:0810.3194 [nucl-th].

[23] see, for example: J. Raufeisen, J. C. Peng and G. C. Nayak, Phys. Rev. D 66, 034024 (2002) arXiv:hep-ph/0204095; R. Baier, A. H. Mueller and D. Schiff, Nucl. Phys. A 741, 358 (2004) arXiv:hep-ph/0403201; F. Gelis and J. JalilianMarian, Phys. Rev. D 76, 074015 (2007) arXiv:hep-ph/0609066], and references therein.

[24] X. F. Guo, Phys. Rev. D 58, 036001 (1998) arXiv:hep-ph/9711453]; J. W. Qiu and X. Zhang, Phys. Lett. B 525, 265 (2002) arXiv:hep-ph/0109210; Z. B. Kang and J. W. Qiu, Phys. Rev. D 77, 114027 (2008) arXiv:0802.2904 [hep-ph]].

[25] see, for example: X. F. Guo and J. W. Qiu, Phys. Rev. D 53, 6144 (1996) arXiv:hep-ph/9512262; S. Peigne, Phys. Rev. D 66, 114011 (2002) arXiv:hep-ph/0206138; R. J. Fries, Phys. Rev. D 68, 074013 (2003) arXiv:hep-ph/0209275; B. Z. Kopeliovich, J. Raufeisen, A. V. Tarasov and M. B. Johnson, Phys. Rev. C 67, 014903 (2003) arXiv:hep-ph/0110221, and references therein.

[26] see, for example: S. Turbide, C. Gale, D. K. Srivastava and R. J. Fries, Phys. Rev. C 74, 014903 (2006) arXiv:hep-ph/0601042; H. van Hees and R. Rapp, Nucl. Phys. A 806, 339 (2008) arXiv:0711.3444 [hep-ph]]; B. Kämpfer and O. P. Pavlenko, Phys. Lett. B 391, 185 (1997). See also: N. Armesto et al., J. Phys. G 35, 054001 (2008) arXiv:0711.0974 [hep-ph]]; F. Arleo et al., arXiv:hep-ph/0311131, P. Aurenche, arXiv:hep-ph/0610218, and references therein.

[27] K. J. Eskola, V. J. Kolhinen and C. A. Salgado, Eur. Phys. J. C 9, 61 (1999) arXiv:hep-ph/9807297; K. J. Eskola, 
V. J. Kolhinen and P. V. Ruuskanen, Nucl. Phys. B 535, 351 (1998) arXiv:hep-ph/9802350.

[28] L. Frankfurt, V. Guzey and M. Strikman, Phys. Rev. D 71, 054001 (2005) arXiv:hep-ph/0303022.

[29] M. Hirai, S. Kumano and M. Miyama, Phys. Rev. D 64, 034003 (2001) arXiv:hep-ph/0103208; M. Hirai, S. Kumano and T. H. Nagai, Phys. Rev. C 76, 065207 (2007) arXiv:0709.3038 [hep-ph]].

[30] D. de Florian and R. Sassot, Phys. Rev. D 69, 074028 (2004) arXiv:hep-ph/0311227.

[31] K. J. Eskola, H. Paukkunen and C. A. Salgado, arXiv:0802.0139 [hep-ph].

[32] R. Vogt, Phys. Rev. C 70, 064902 (2004); K. J. Eskola, Z. Phys. C 51, 633 (1991).

[33] D. Peressounko [PHENIX Collaboration], Nucl. Phys. A 783, 577 (2007) arXiv:hep-ex/0609037.

[34] C. Klein-Boesing [PHENIX Collaboration], J. Phys. G 35, 044026 (2008) arXiv:0710.2960 [nucl-ex]].

[35] M. L. Miller, K. Reygers, S. J. Sanders and P. Steinberg, Ann. Rev. Nucl. Part. Sci. 57, 205 (2007) arXiv:nucl-ex/0701025; S. S. Adler et al. [PHENIX Collaboration], Phys. Rev. Lett. 91, 072301 (2003) arXiv:nucl-ex/0304022. 